\title{
Bifurcation analysis in a silicon neuron
}

\author{
F. Grassia ${ }^{1}$, T. Levi ${ }^{1}$, S. Saighi ${ }^{1}$ and T. Kohno ${ }^{2}$ \\ ${ }^{1}$ Laboratoire IMS, UMR CNRS 5218, Université de Bordeaux, Talence, France \\ ${ }^{2}$ IIS University of Tokyo, Japan \\ ${ }^{1}$ filippo.grassia@ims-bordeaux.fr
}

\begin{abstract}
In this paper, we describe an analysis of the nonlinear dynamical phenomenon associated with a silicon neuron. Our silicon neuron integrates Hodgkin-Huxley $(\mathrm{HH})$ model formalism, including the membrane voltage dependency of temporal dynamics. Analysis of the bifurcation conditions allow us to identify different regimes in the parameter space that are desirable for biasing our silicon neuron. This approach of studying bifurcations is useful because it is believed that computational properties of neurons are based on the bifurcations exhibited by these dynamical systems in response to some changing stimulus. We describe numerical simulations and measurements of the Hopf bifurcation which is characteristic of class 2 excitability in the HH model. We also show a phenomenon observed in biological neurons and termed excitation block. Hence, by showing that this silicon neuron has similar bifurcations to a certain class of biological neurons, we can claim that the silicon neuron can also perform similar computations.
\end{abstract}

Keywords: Silicon Neuron, Hopf bifurcation, Hodgkin-Huxley equations, neuromorphic engineering.

\section{INTRODUCTION}

Neuromorphic engineering is a research field where microelectronics meets biology. The link between both is done by computational neurosciences which model and emulate a part of brain activity. Neuromorphic systems emulate biological structures in the hope of retaining its remarkable computational efficiency. Analysis of the bifurcation is useful because it is believed that computational properties of neurons are based on the bifurcations exhibited by these dynamical systems in response to some changing stimulus [1] [2]. These artificial systems would be useful to neuroscientists for exploring neural computation. Contrarily to computer simulation, analog circuits are inherently suitable for simulating differential equations in real time and in parallel [3]. The hardware simulation further facilitates the building of a hybrid network connecting artificial and biological neurons to create a real-time loop or can be even used to replace nonfunctional neurons as a prosthesis.

Pioneering work in modeling the dynamics of a biological neuron was done by Hodgkin and Huxley $(\mathrm{HH})$ [4]. In the most detailed family of neuron models, known as conductance-based models, ionic and synaptic currents charge and discharge a capacitor representing the neuron membrane. All of these models find their origins in the $\mathrm{HH}$ formalism. Each ionic channel (sodium, potassium, calcium, etc.) is represented by a time and voltage dependent conductance: this electrophysiological description makes these models particularly well-suited to an implementation involving analog electronics [5]. The main advantage of this formalism is that it relies on biophysically realistic parameters and describes individual ionic and synaptic conductances for each neuron in accordance with the dynamics of ionic channels.

Hodgkin classified biological neurons mainly into two classes according to their response properties to a sustained current stimulus [6]. The cells that show Class 1 neural excitability can fire with an arbitrarily low frequency by applying a sufficiently close-to-threshold current. The frequency increases monotonically as the current increase. The cells that show Class 2 neural excitability transitioned from silence to firing at an arbitrary nonzero frequency. Furthermore, the Class 1 characteristic is can be associated with a saddle-node bifurcation at the transition from silence to spiking. As shown by bifurcation analysis, the Class 2 excitability is observed when the resting state loses its stability via an Hopf-bifurcation.

The Hodgkin-Huxley model belongs to Class 2. Its dynamical properties with a constant stimulus have been studied extensively [7] [8], and these have shown that the Class 2 neural excitability of the HH model is generated by Hopf bifurcations.

Innovative work in modeling of a simplified silicon neuron that exhibits HH type dynamics was done in [9] and in [10]. On the other hand, some designs have faithfully replicated full Hodgkin-Huxley dynamics, resulting in large footprints for each neuron [5]. We implement in our chip the $\mathrm{HH}$ model with an approximation: we use a fixed time constant to reduce the silicon area required by the neuron implementation in the chip. In this paper we present an analysis of the nonlinear dynamical phenomenon associated 
with our silicon neuron. We show a Hopf bifurcation which is characteristic of class 2 excitability in the $\mathrm{HH}$ model in our approximated model. We also show a phenomenon observed in real neurons and termed excitation block.

\section{HH MODEL IN VLSI}

\subsection{The Hodgkin-Huxley Formalism}

We chose to implement neuron model following the Hodgkin-Huxley formalism. Electrical activity of a neuron is the consequence of the diffusion of different ionic species through its membrane. The HH formalism provides a set of equations and an equivalent electrical circuit which describes conductance phenomena.

The current flowing across the membrane is integrated on the membrane capacitance, according to expression (1):

$$
\underset{C_{1}}{\mathrm{dt}}=\boldsymbol{r}_{\mathbf{L}}+\mathbf{H}_{\mathbf{S}}
$$

where $V_{M}$ is the membrane potential, $C_{M}$ is the membrane capacitance, and $\mathrm{I}_{\mathrm{S}}$ is a stimulation or synaptic current. $\mathrm{I}_{\mathrm{I}}$ is the current for a given channel type and its associated equation is:

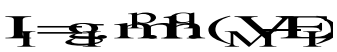

where $\mathrm{g}_{\mathrm{I}}$ is the maximum conductance; $\mathrm{m}$ and $\mathrm{h}$ represent the activation and inactivation terms, respectively. They are dynamic functions, which describe the permeability of membrane channels to its specific ion. $E_{I}$ is the ion-specific reverse potential and $\mathrm{p}$ and $\mathrm{q}$ are integers.

According to the first order differential equation (3), $\mathrm{m}$ relaxes back towards its associated steady-state value $\mathrm{m}_{\infty}$, which is a sigmoid function of $\mathrm{V}_{\mathrm{M}}$ (4). The time constant for convergence is $\tau_{\mathrm{m}}$ which is also voltage dependent of the membrane voltage $\mathrm{V}_{\mathrm{M}}$.
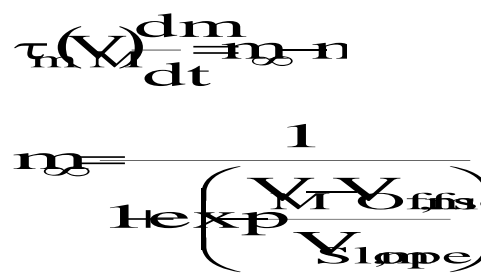

In (4), $V_{\text {Offset,m }}$ and $V_{\text {Slope, } m}$ are the offset and the slope of the activation sigmoid respectively. The inactivation parameter $\mathrm{h}$ follows identical equations, except for the sign inside the brackets, which is positive.

The HH model describes sodium, potassium and leakage channels, with $\mathrm{p}=3$ and $\mathrm{q}=1 ; \mathrm{p}=4$ and $\mathrm{q}=0 ; \mathrm{p}=$ 0 and $q=0$ respectively, in expression (2). These channels are responsible for action potential generation. For more complex activity patterns, such as bursting or the discharge of action potentials with adaptation phenomena, additional channels such as slow potassium $(\mathrm{p}=1$ and $\mathrm{q}=0)$ and $\mathrm{L}$ Type calcium for bursting ( $p=2$ and $q=1$ ) have to be taken into account. Our chip has a large range of validity domains for the parameter to reproduce different kind of neurons like: Fast Spiking (FS), Regular Spiking (RS), Intrinsically Bursting (IB), and Low Threshold Spiking (LTS) [11]. Although these HH-type models sometimes exhibit much more complex dynamics than the original $\mathrm{HH}$ model, they share common nonlinear characteristics and dynamics with the $\mathrm{HH}$ in many aspects.

\subsection{The model dedicated to our integrated circuit}

Our system is composed of our most recent chip called Galway and the dedicated board named Ekerö. This chip includes analog operators for the computation of the $\mathrm{HH}$ formalism, and for the construction of neural networks, multi-synapses that consist of gathering all synaptic inputs in one electronic input. We implement in our chip the $\mathrm{HH}$ model with an approximation: we use a fixed time constant in (3). We made this choice to reduce the size of the chip. So the only difference between the model for the VLSI and the HH model presented in [12] is the approximation used for the gating variable.

\subsection{Hopf bifurcation}

The Hopf bifurcation theory [8] asserts that if a parameterized system possesses an equilibrium point and two of the eigenvalues of the Jacobian matrix of the system linearized around the equilibrium point are conjugate pure imaginary numbers and the others have negative real parts, one of the following bifurcations takes place as the parameter changes:

1) A bifurcation from a stable equilibrium point to an unstable equilibrium point with a stable limit cycle around it; or a bifurcation with the opposite direction. We refer to this bifurcation as supercritical.

2) A bifurcation from an unstable equilibrium point to a stable equilibrium point with an unstable limit cycle around it; or a bifurcation with the opposite direction. We refer to this bifurcation as subcritical.

\subsection{Simulation}

To validate our approximation we used continuation of solutions in AUTO, an open source mathematical package that can produce bifurcation curves for equilibria as well as for periodic orbits, to get the complete bifurcation diagram of the system. We compare the simulations between $\mathrm{HH}$ based model and our implemented HH model. 


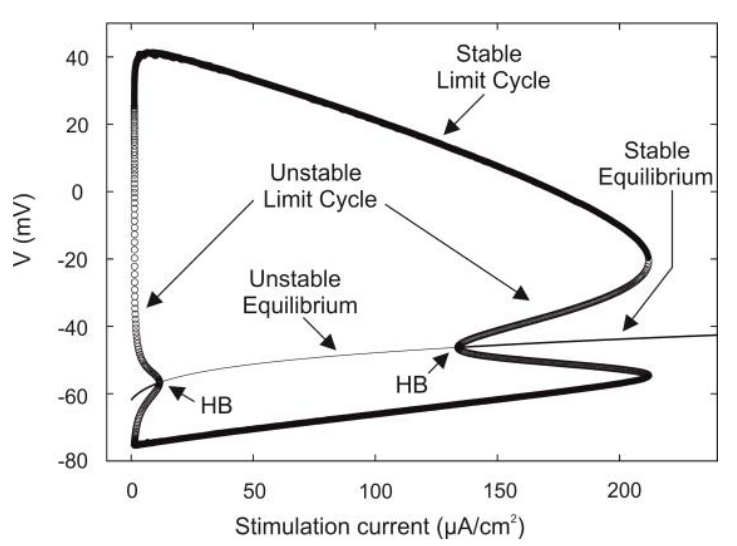

Fig. 1. Bifurcation diagram for our approximated model.

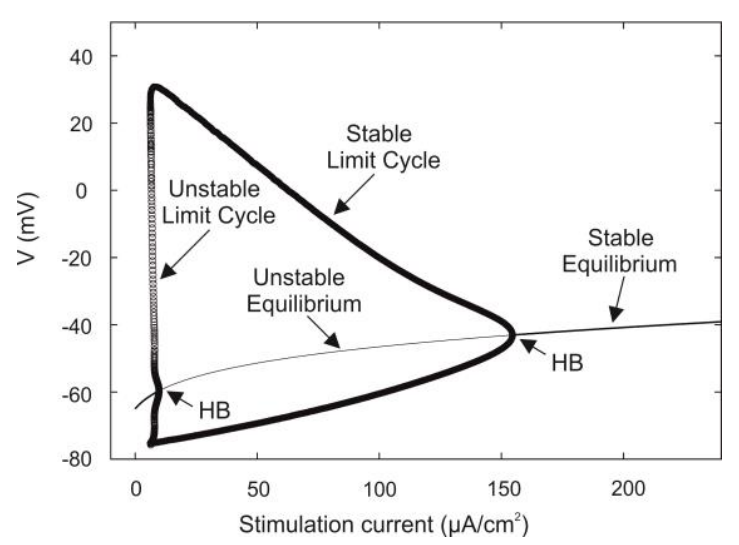

Fig. 2. Bifurcation diagram for $\mathrm{HH}$ model.

Fig. 1 shows the resulting bifurcation diagram of our $\mathrm{HH}$ approximated model where solid thick and solid thin curves represent stable and unstable equilibria respectively while solid and dashed circles denote stable and unstable limit cycles. It can be seen that the limit cycles are born initially through a fold bifurcation of cycles. Both the Hopf bifurcations are subcritical as they involve an unstable limit cycle. The amplitude of the stable limit cycle keeps on reducing until it coalesces with the unstable limit cycle in another fold bifurcation. The resting state loses stability via the subcritical Hopf bifurcation at the first point HB in Fig. 1. At the second point HB in Fig. 2, a stable limit cycle and an unstable one arise via the fold bifurcation. Bifurcation diagram in Fig. 1 illustrate how the repetitive firing emerges when we apply sustained stimulus current. Stable limit cycle corresponds to repetitive firing.

For a comparison with $\mathrm{HH}$ model, its bifurcation is also shown in Fig. 2. The Hopf bifurcation for smaller current is subcritical. The limit cycle appears by a fold bifurcation and disappears by a supercritical Hopf bifurcation. The reduction in amplitude before the subcritical Hopf bifurcation is similar to the Fig. 1. It can be seen in Fig. 2 that the limit cycle is born by a fold bifurcation but terminates in a supercritical Hopf bifurcation. The bifurcation diagrams shown in Fig. 1 and Fig. 2 share the topology in a biologically meaningful range of stimulus current. The qualitative nature of the plots (Fig. 1 and Fig. 2 ) is similar as the reduction in amplitude of the limit cycle before its disappearance is present in both pictures.

\section{RESULTS IN VLSI}

\subsection{Firing rate}

In Fig. 1, the system state jumps to the stable limit cycle when the stimulus current exceeds the Hopf bifurcation point. In this case the firing frequency cannot be zero at the bifurcation point for the Class 2. Steps of input current are important type of stimulus typically used to characterize the behavior of neuron models. The resulting spike frequencies for different values of stimulation current are plotted in Fig. 3. This figure shows a behavior typical of class 2 neurons where the spiking frequency does not tend zero upon reduction of the input current. In other word, the disappearance of the limit cycle is not associated with its frequency reducing to zero like in class 1 neurons.

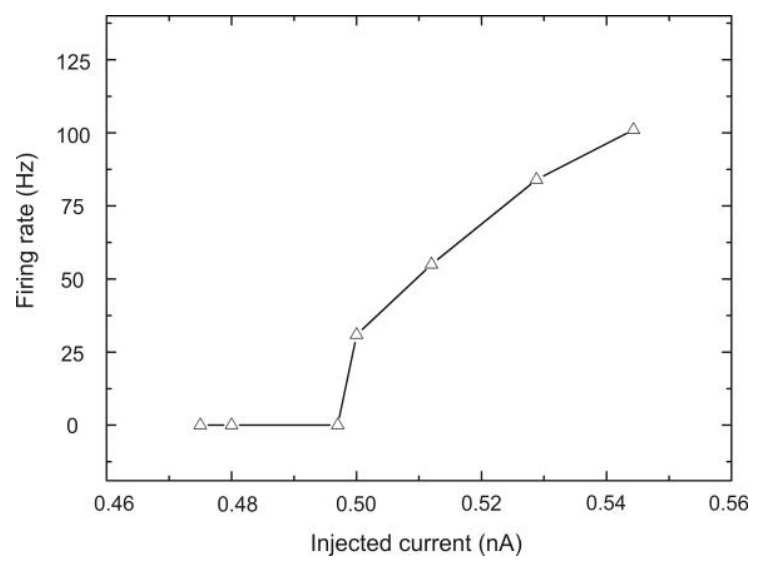

Fig. 3. Hardware measurements of the frequency versus stimulation current curves of our silicon neurons.

\subsection{Effect of current impulses}

Fig. 4 shows the effect of current impulses of different values in our silicon neuron. Larger current pulses shifts the initial condition beyond the unstable limit cycle leading to spontaneous oscillations. We used four different current pulses of increasing amplitude to stimulate our silicon neuron. It can be seen in Fig. 4 that for the first two case the neuron come back to resting state and in the third and fourth case it start the oscillations. This demonstrates the 
existence of the threshold that in our silicon neuron is 0.5 nA.

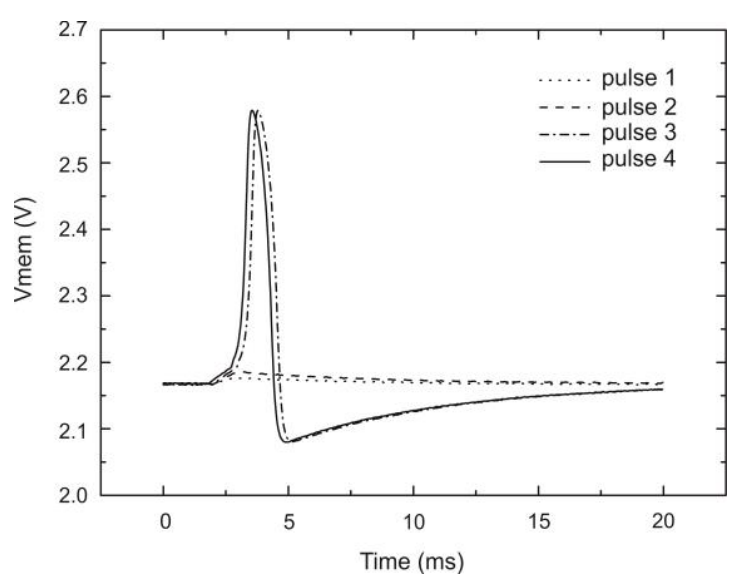

Fig. 4. Hardware neuron membrane voltage depending on current impulses.

\subsection{Excitation block}

To prove that the silicon neuron reproduces similar dynamical properties of biological neuron, we also explored the phenomenon known in biology and termed excitation block. In this case, there is the cessation of repetitive spiking as the amplitude of the stimulus current increases. The equilibrium regains stability through a Hopf bifurcation which may be subcritical or supercritical. To observe this phenomenon a slowly increasing ramp of current was injected. In Fig. 5 is showed that for much larger value of the stimulus the equilibrium becomes stables again.

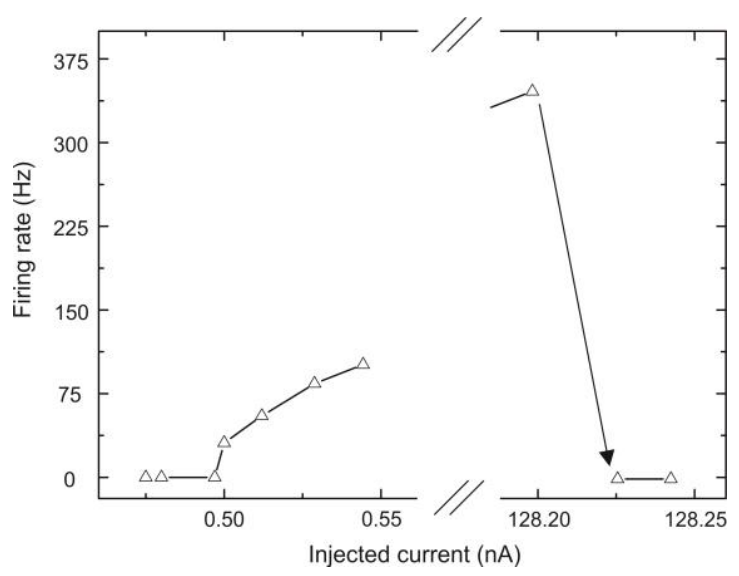

Fig. 5. Measured data of the excitation block where the input current is slowly increased.

\section{CONCLUSION}

In this paper, we described an analysis of the nonlinear dynamical phenomenon associated with a silicon neuron. We implemented an approximated $\mathrm{HH}$ model using fixed time constant. We showed numerical simulations of the Hopf bifurcation which is characteristic of Class 2 excitability in the $\mathrm{HH}$ model. We compared the results with the $\mathrm{HH}$ model showing that our model shares the dynamics with the full $\mathrm{HH}$ model. We also showed the firing rate and then the phenomenon observed in real neurons and termed excitation block. Hence, by showing that this silicon neuron has nonlinear dynamical phenomenon similar to a certain class of biological neurons, we can claim that the silicon neuron can also perform similar computations.

\section{ACKNOWLEDGMENTS}

This project was partly supported by funding under the Seventh Research Framework Program of the European Union (FP7-PEOPLE-ITN-2008) under the Grant $n^{\circ} 237955$ (FACETS-ITN).

\section{REFERENCES}

[1] Izhikevich EM (2000), Neural excitability, spiking and bursting. IJBC, vol.10, pp. 1171-1266.

[2] Rinzel J, Ermentrout GB (1989), Analysis of Neural excitability and oscillations. Methods in Neural Engineering, Koch C and Segev I, Eds, Cambridge.

[3] Chua LO, Roska T, Kozek et al (1996), CNN universal chips crank up the computing power.

IEEE Circuits Devices Mag., vol. 12, pp. 18-28.

[4] Hodgkin, AL, Huxley AF (1952). A quantitative description of membrane current and its application to conduction and excitation nerve. Journal of physiology, vol.117, pp. 500-544.

[5] Simoni MF, Cymbalyuk GS, Sorensen ME et al(2004), A multiconductance silicon neuron with biologically matched dynamics. IEEE Transactions on Biome-dical Engineering, vol. 51, pp. 342-354.

[6] Hodgkin AL (1948), The local electric changes associated with repetitive action in a non medullated axon. Journal of physiology, vol. 107, pp. 165-181.

[7] Hassard B (1978), Bifurcation of periodic soluti-ons of the Hodgkin-Huxley model of the squid giant axon. J. Theor. Biol., vol. 71, pp. 401-420.

[8] Hassard B, Kazarinoff ND, Wan YH (1981), Theory and Applications of Hopf Bifurcation. Cambrid-ge, U.K.: Cambridge Univ. Press.

[9] Kohno T, Aihara K (2005), A MOSFET - basedmodel of a class 2 nerve membrane. IEEE Transac. onNeural Networks, vol. 16, no. 3, pp. 754-773.

[10] Basu A, Petre C, Hasler P (2010), Dynamics and Bifurcations in a Silicon Neuron. IEEE Tr. on Biomedical Circuits and Systems vol. 4, pp. 320-328.

[11] Grassia F, Buhry L, Lévi T et al (2011), Tuna-ble neuromimetic integrated system for emulating corti-al neuron models. Front. Neurosci. 5:134.

[12] Hansel D, Mato G, Meunier C (1993), Phase dynamics for weakly coupled Hodgkin-Huxley neurons.Europhys. Lett, vol. 23, pp. 367-372. 